\title{
The Physical State Space of Quantum Electrodynamics
}

\author{
Detlev Buchholz \\ II. Institute of Theoretical Physics, University of Hamburg, D-2000 Hamburg, \\ Federal Republic of Germany
}

\begin{abstract}
Starting from the fact that electrically charged particles are massive, we derive a criterion which characterizes the state space of quantum electrodynamics. This criterion clarifies the special role of the electric charge amongst the uncountably many superselection rules in quantum electrodynamics and provides a basis for a general analysis of the infrared problem. Within this framework we establish the existence of asymptotic electromagnetic fields in all charge-sectors, find a general characterization of infra-particles and introduce a notion of asymptotic completeness.
\end{abstract}

\section{Introduction}

In striking contrast to the excellent experimental confirmation of quantum electrodynamics, the understanding of its conceptual foundations is still only of a rather qualitative nature. Besides the well-known infrared problems which one encounters in the interpretation of physical states at asymptotic times [1], the even more fundamental problem of defining a physical state space has not yet been solved in a satisfactory manner. It is the aim of the present contribution to clarify this point and thereby to provide a basis for a general discussion of the structure of quantum electrodynamics.

There are two related problems which one encounters in the definition of a state space. First, there exists the well-known difficulty that physical states carrying an electric charge cannot be constructed by applying local field operators to the vacuum state. This fact can be traced back to Gauss' law which implies that the electric charge of a particle can be determined by measuring the total electric flux through an arbitrarily large sphere surrounding the particle $[2,3]$.

The second complication, which is less frequently noticed although it has the same physical origin, consists in the fact that there exist uncountably many superselection sectors in quantum electrodynamics. This may be seen from the following heuristic argument: choosing some Lorentz system, the observable $\phi$ 
measuring the asymptotic electric flux-distribution in spatial directions $\mathbf{n},|\mathbf{n}|=1$ is given by

$$
\phi(\mathbf{n})=\lim _{r \rightarrow \infty} r^{2} \cdot \mathbf{n} \cdot \mathbf{E}(r \cdot \mathbf{n}) .
$$

Here $\mathbf{E}$ is the suitably regularized electric field operator, and it is tacitly assumed that the limit can be defined on the states of interest. $\phi$ is a classical observable because it commutes with all local observables due to the principle of locality. Therefore it is represented in each superselection sector by some $c$-number function $\mathbf{n} \rightarrow \varphi(\mathbf{n})$ describing the asymptotic electric flux-distribution of the states in that sector [24].

Now the only physical constraint imposed on the functions $\varphi$ is that the total flux $\int d \omega \varphi(\mathbf{n})$ must be a multiple of the electric charge. This indicates that there exists a tremendous number of superselection sectors in quantum electrodynamics corresponding to a variety of asymptotic flux-distributions $\varphi$. In particular, one can generate a continuum of states belonging to sectors with different asymptotic flux-distributions $\varphi_{A}$ by acting on any charged state with all Lorentz transformations $\Lambda$. (The latter fact also explains the breakdown of Lorentz covariance in sectors describing charged states [4].)

In the conventional field-theoretic treatment of quantum-electrodynamics one simply ignores this abundance of sectors and selects in some arbitrary way a convenient subset of states. In the Coulomb-gauge [5], for example, this is done by constructing a nonlocal charge-carrying field operator which generates states from the vacuum with a fixed asymptotic flux-distribution (usually one chooses a spherically symmetric one). The resulting Hilbert-space consists then of a countable number of superselection-sectors which can be distinguished by their electric charge.

It is less obvious how such a selection of states is achieved in the Gupta-Bleuler formalism [3]. There one constructs with the help of the local gauge-fields an indefinite metric space of unphysical vectors, which are expected to approximate the physical states in a suitable topology. Since the choice of this topology is highly ambiguous, it is not quite clear which physical sectors are actually obtained by this procedure. But the resulting physical Hilbert-space is always separable, so that it also contains an at most countable number of superselection-sectors with a discrete weight.

Despite the fact that these field-theoretic settings do not include all superselection sectors, they cover all situations of physical interest. In fact it follows from the well-known "particle behind the moon"-argument of Haag and Kastler that even the states in a single superselection-sector would be sufficient to deduce all the physically relevant information [6]. But the conventional field-theoretic settings are not a very convenient frame if one wants to express the fact that there exist sectors in quantum electrodynamics with almost arbitrary asymptotic fluxdistributions. Since we want to exploit this important feature which allows us to choose the localization properties of charged states within certain limitations, we will use here the more flexible Haag-Kastler framework of local quantum theory [6]. In this framework a unified treatment of all superselection sectors of quantum electrodynamics is possible! 
Using the fact that the charged particles are massive, we will introduce in Sect. 2 an equivalence relation between sectors of a given electric charge. The resulting concept of charge-classes is used in Sect. 3 to select those charged states which have the best possible localization properties with respect to the vacuum. We propose to consider this set of states as the physical state space of quantum electrodynamics. In Sect. 4 we will show that one can define on these states incoming and outgoing electromagnetic fields as limits of local observables. Using this result we will also obtain some information on the asymptotic observables which are associated with the charged particles. In particular, we will find a general characterization of infra-particles and introduce a notion of asymptotic completeness which applies to quantum electrodynamics. With this input, we can show in Sect. 5, that the sectors within a charge-class only differ by radiation fields which are induced by an infinite number of incoming, respectively outgoing, lowenergy photons (infrared-clouds). This result establishes the consistency of the underlying physical ideas.

We conclude this introduction with a list of assumptions. The basic objects in our analysis are the local observables, which according to the fundamental work of Haag and Kastler are known to embody all the relevant physical information. In quantum electrodynamics these observables are the electromagnetic field $F_{\mu \nu}$ and the electric current $j_{\nu}=\partial^{\mu} F_{\mu \nu}$. In order to avoid the discussion of domain problems we assume that these field-operators smoothed out with suitable testfunctions generate a net $\mathcal{O} \rightarrow \mathfrak{U}(\mathcal{O})$ of $C^{*}$-algebras indexed by the bounded regions $\mathcal{O}$ of Minkowski space. Thus each $\mathfrak{U}(\mathcal{O})$ may be regarded as the algebra of all observables which can be measured within $\mathcal{O}$. For unbounded regions $\mathscr{R}$, the algebras of observables $\mathfrak{U}(\mathscr{R})$ are defined as the $C^{*}$-inductive limits of the local algebras $\mathfrak{H}(\mathcal{O})$ with $\mathcal{O} \subset \mathscr{R}$,

$$
\mathfrak{A}(\mathscr{R})=\bigcup_{\mathscr{O} C \mathscr{R}}^{C^{\star}} \mathfrak{A}(\mathcal{O}),
$$

and $\mathfrak{U}=\mathfrak{U}\left(\mathbb{R}^{4}\right)$ denotes the algebra of all local observables. We recall that observables in spacelike separated regions commute (locality)

$$
\left[\mathfrak{U}\left(\mathcal{O}_{1}\right), \mathfrak{U}\left(\mathcal{O}_{2}\right)\right]=0 \text { if } \quad \mathcal{O}_{1} \subset \mathcal{O}_{2}^{\prime}
$$

and that the Poincare transformations $L$ induce automorphisms $\alpha_{L}$ of $\mathfrak{A}$ which respect the local structure of the net (covariance)

$$
\alpha_{L}(\mathfrak{U}(\mathcal{O}))=\mathfrak{U}(L \cdot \mathcal{O})
$$

The physical states in quantum electrodynamics are distinguished by the fact that they induce representations $(\pi, \mathscr{H})$ of the algebra $\mathfrak{U}$ on a separable Hilbertspace $\mathscr{H}$ which are invariant with respect to the space-time translations $x=(t, \mathbf{x})$. This means that there exists a strongly continuous unitary representation $x \rightarrow U(x)$ of the translations on $\mathscr{H}$ such that the operators $U(x)$ implement the action of $\alpha_{x}$

$$
\pi\left(\alpha_{x}(A)\right)=U(x) \cdot \pi(A) \cdot U(x)^{-1} \text { for } A \in \mathfrak{A} .
$$

Moreover, the generators $P=\left(P_{0}, \mathbf{P}\right)$ of $U(x)$ satisfy the relativistic spectrum condition, i.e.

$$
\operatorname{sp} \mathscr{U} \subset\left\{p: p^{2} \geqq 0, p_{0} \geqq 0\right\} \text {. }
$$


If the representation $\pi$ is reducible, then the operators $U(x)$ are not fixed by these conditions. It can be shown, however, that it is always possible to find operators $U(x) \in \pi(\mathfrak{A})^{-}$such that the spectrum of the generators $P$ has a Lorentz invariant lower boundary ${ }^{1}$ in each factorial subrepresentation of $\pi$ [7], and this property fixes them uniquely. We will call representations exhibiting these properties positive energy representations.

Amongst the positive energy representations in quantum electrodynamics there exists in particular the irreducible vacuum representation $\left(\pi_{0}, \mathscr{H}_{0}\right)$ of $\mathfrak{A}$. We assume that there is a continuous unitary representation $L \rightarrow U(L)$ of the full Poincaré group on $\mathscr{H}_{0}$ which implements the automorphisms $\alpha_{L}$ and leaves invariant the (up to a phase unique) vector $\Omega \in \mathscr{H}_{0}$ representing the vacuum state. Moreover, there is a subspace $\mathscr{H}_{0}^{(1)} \subset \mathscr{H}_{0}$, describing states of a single photon, on which the Poincaré transformations $U(L)$ act like a direct sum of irreducible representations of zero mass.

We mention as an aside, that starting from a local, covariant field such as $F_{\mu \nu}$, there is a canonical way of constructing in the vacuum representation a net $\mathcal{O} \rightarrow \mathfrak{U}(\mathcal{O})$ of local algebras. This net satisfies the above assumptions and in addition duality [8], a powerful maximality condition invented by Haag [9]. It is therefore natural to consider the vacuum representation as the defining representation of $\mathfrak{U}$.

\section{Charge-Classes}

A superselection sector is defined as a class of unitarily equivalent irreducible representations of $\mathfrak{A}$, where we restrict our attention to positive energy representations as defined in the Introduction. In view of the preceding discussion we must be prepared for a tremendous number of such sectors corresponding to different asymptotic electric flux-distributions, and there arises the question as to what distinguishes the electric charge from the other superselection rules in quantum electrodynamics.

An answer to this question can easily be given in physical terms: the electric charge is tied to massive particles which move with a velocity which is less than the velocity of light. It is therefore possible to determine the total electric charge of a state by measurements in proper subregions of Minkowski-space, such as the lightcone $V_{+}$. This is plausible if one notices that all charged particles which are described by a state will eventually enter that cone, provided they are not annihilated in pairs. On the other hand it is not possible to determine the asymptotic electric flux-distribution in $V_{+}$, because photons coming from the remote past cannot be observed in that cone, but they will in general affect the flux-distribution.

The fact that the electric charge of a state can be determined in a lightcone, but not its sector, indicates that the positive energy representations of $\mathfrak{A}$ can be combined into classes of representations whose restrictions to the subalgebras $\mathfrak{A}\left(V_{+}+a\right)$ are equivalent for all $a \in \mathbb{R}^{4}$. Each such class should contain representations of a fixed electric charge but quite arbitrary asymptotic fluxdistributions. We will verify this assertion in the following analysis.

1 This result follows only from the spectrum condition and locality, so that it can be applied in a situation where the Lorentz transformations are not unitarily implemented 
Given any positive energy representation $(\pi, \mathscr{H})$ of $\mathfrak{A}$ we consider its center, $3=\pi(\mathfrak{U})^{-} \cap \pi(\mathfrak{U})^{\prime}$, and the center of its restriction to $\mathfrak{U}\left(V_{+}+a\right)$ where $a \in \mathbb{R}^{4}$ is fixed, $3_{+}=\pi\left(\mathfrak{U}\left(V_{+}+a\right)\right)^{-} \cap \pi\left(\mathfrak{U}\left(V_{+}+a\right)\right)^{\prime}$. The following lemma is then a consequence of the spectrum condition for the translations $U(x)$ in the representation $\pi$.

Lemma 2.1. i) The elements of $3_{+}$are invariant under arbitrary translations, $U(x) Z U(x)^{-1}=Z$ for $Z \in 3_{+}$.

ii) $3+c 3$.

Proof. Let $n$ be any positive timelike vector. Then it is obvious that $U(t \cdot n) \cdot 3_{+} \cdot U(t \cdot n)^{-1} \subset \mathfrak{Z}_{+}^{\prime}$ for $t \in \mathbb{R}$, so that the von Neumann algebra $\bigvee_{t^{\prime}} U\left(t^{\prime} \cdot n\right) \cdot 3_{+} \cdot U\left(t^{\prime} \cdot n\right)^{-1}$ is Abelian. This algebra is, by construction, stable under the automorphisms induced by the translations $U(t \cdot n)$. On the other hand we know from the spectrum condition (1.6) that the generator of $t \rightarrow U(t \cdot n)$ is positive. It then follows from a theorem of Borchers [10] that the elements of this algebra are pointwise fixed under the action of $U(t \cdot n)$, hence $U(t \cdot n) \cdot Z \cdot U(t \cdot n)^{-1}=Z$ for $Z \in 3_{+}$. This proves the first part of the statement, because the linear span of all positive timelike vectors $n$ is $\mathbb{R}^{4}$. The second part is then an immediate consequence. QED

Since $3_{+} \subset 3$, one can decompose the representation $\pi$ with respect to $3_{+}[11]$,

$$
\pi=\int_{\mathrm{sp} 3+} d \mu(\xi) \pi_{\xi}
$$

and the representations $\pi_{\xi}$ belong (for $\mu$-almost all $\xi$ ) to the class of positive energy representations. Moreover, since $3_{+}=\pi\left(\mathfrak{U}\left(V_{+}+a\right)\right)^{-} \cap \pi\left(\mathfrak{U}\left(V_{+}+a\right)\right)^{\prime}$ does not depend on $a$, it follows that the restrictions of the representations $\pi_{\xi}$ to the algebras $\mathfrak{U}\left(V_{+}+a\right), a \in \mathbb{R}^{4}$ are factorial. It is therefore sufficient to concentrate in the following on such representations.

Now given any two such representations, it is clear that they induce either quasi-equivalent or disjoint representations of the algebras $\mathfrak{U}\left(V_{+}+a\right), a \in \mathbb{R}^{4}$. We shall see that in the present case one can replace in this general alternative the notion of quasi-equivalence by unitary equivalence. At this point it is essential that the model describes massless particles.

This fact should imply that one can define asymptotic electromagnetic fields as LSZ-type of limits of local observables in the representations $(\pi, \mathscr{H})$ of interest. If there is any reasonable way of constructing the outgoing field $F^{\text {out }}$, for example, it should generate a net $\mathcal{O} \rightarrow \mathfrak{F}^{\text {out }}(\mathcal{O})$ of von Neumann algebras on $\mathscr{H}$, which is covariant with respect to the action of the translations $U(x)$ and which has in addition the following properties. (These properties will subsequently be derived in the frame of the infrared minimal representations, cf. Theorem 4.1 below.)

i) $\mathfrak{F}^{\text {out }}(\mathcal{O}) \subset \pi\left(\mathfrak{A}\left(V_{+}+a\right)\right)^{-}$if $\mathcal{O} \subset V_{+}+a$

ii) $\mathfrak{F}^{\text {out }}(\mathcal{O}) \subset \pi\left(\mathfrak{U}\left(V_{+}+b\right)\right)^{\prime}$ if $\mathcal{O} \subset V_{-}+b$

iii) $\bigcup_{\mathscr{O}}^{C^{*}} \mathfrak{F}^{\text {out }}(\mathcal{O})=\mathfrak{F}^{\text {out }}$ has a cyclic vector in $\mathscr{H}$.

The first property follows from the physical meaning of $F^{\text {out }}$ and the second one amounts to Huygens' principle [12]. It is only the third property which 
requires some explanations. Since $\mathfrak{F}^{\text {out }}$ is generated by a free field, it is a realization of the algebra of canonical commutation relations, and it is therefore clear that $\mathfrak{F}^{\text {out }-}$ does not have any normal tracial state. Thus the von Neumann algebra $\mathfrak{F}^{\text {out }}$ - is properly infinite, and since also $1 \in \mathfrak{F}^{\text {out }-}$ it follows [11, Theorem 2.2.4] that there exist infinitely many isometries $V_{i} \in \mathfrak{F}^{\text {out }}$,,$i \in \mathbb{N}$ with orthogonal ranges, i.e. $V_{i}^{*} V_{j}=\delta_{i j} \cdot 1$. Hence one must only choose some orthonormal basis $\left\{\Phi_{i}\right\}_{i \in \mathbb{N}}$ in $\mathscr{H}$, and the vector $\Phi=\sum_{1}^{\infty} 2^{-i} \cdot V_{i} \Phi_{i}$ is then clearly cyclic for $\mathfrak{F}^{\text {out }}$.

After these preparations, we can now continue our analysis of those positive energy representations $\pi$ for which $\pi\left(\mathfrak{U}\left(V_{+}+a\right)\right)^{-}$is a factor. The following lemma, where we exploit the anticipated structural properties of $\mathfrak{F}^{\text {out }}$, is the analogue of a result of Borchers [13].

Lemma 2.2. Let $E \in \pi\left(\mathfrak{U}\left(V_{+}+a\right)\right)^{\prime}, E \neq 0$ be a projection. Then, for some $b \in \mathbb{R}^{4}$, there exists an isometry $V \in \pi\left(\mathfrak{U}\left(V_{+}+b\right)\right)^{\prime}$ such that $V^{*} \cdot V=1$ and $V \cdot V^{*}=E$.

Proof. Let $b$ be such that $\left(\bar{V}_{+}+b\right) \subset\left(V_{+}+a\right)$. Then it suffices to establish the existence of a vector $\Phi \in \mathscr{H}$ such that $\Phi$ is cyclic and $E \cdot \Phi$ is separating for the algebra $\pi\left(\mathfrak{U}\left(V_{+}+b\right)\right)^{-}$. Since in that case there exists another cyclic vector $\Psi \in \mathscr{H}$ for which [11, Theorem 2.7.9]

$$
(E \Phi, B \cdot E \Phi)=(\Psi, B \Psi) \quad \text { if } \quad B \in \pi\left(\mathfrak{U}\left(V_{+}+b\right)\right)^{-},
$$

and it is then easy to verify that the isometry $V$ defined by

$$
V \cdot B \Psi=B E \Phi \quad \text { for } \quad B \in \pi\left(\mathfrak{U}\left(V_{+}+b\right)\right)^{-}
$$

has the desired properties.

For the construction of the vector $\Phi$ we pick a region $\mathcal{O}$ such that its closure $\overline{\mathcal{O}} \subset\left(V_{+}+a\right) \cap\left(V_{-}+b\right)$ and a vector $\bar{\Phi} \in \mathscr{H}$ which is cyclic for $\mathfrak{F}^{\text {out }-}$. Now since the spectrum condition holds for $U(x)$ and since $\mathfrak{F}^{\text {out }}$ - is stable under the automorphisms induced by $U(x)$, there exists a representation of the translations $\bar{U}(x) \in \mathfrak{F}^{\text {out }-}$ satisfying the spectrum condition and inducing the same automorphism on $\mathfrak{F}^{\text {out }-}$ as $U(x)[10]$. Hence the vector $\Phi=e^{-\bar{H}} \cdot \bar{\Phi}$, where $\bar{H}$ denotes the positive generator of the time-translations $t \rightarrow \bar{U}(t)$, is also cyclic for $\mathfrak{F}^{\text {out }- \text {. }}$ Moreover, $\Phi$ is an analytic vector for $\bar{H}$, therefore it follows from the ReehSchlieder theorem [14] that $\Phi$ is cyclic for each subalgebra $\bigcup_{x^{\prime} \in \mathcal{N}} U\left(x^{\prime}\right) \cdot \mathfrak{F}^{\text {out }}(\mathcal{O}) \cdot U\left(x^{\prime}\right)^{-1} \subset \mathfrak{F}^{\text {out }}$ if $\mathscr{N}$ is an open region of $\mathbb{R}^{4}$.

Now according to the properties of the net $\mathcal{O} \rightarrow \mathfrak{F}^{\text {out }}(\mathcal{O})$ one has $U(x) \cdot \mathscr{F}^{\text {out }}(\mathcal{O}) \cdot U(x)^{-1} \subset \pi\left(\mathfrak{U}\left(V_{+}+b\right)\right)^{-}$for sufficiently large timelike $x$, hence $\Phi$ is cyclic for $\pi\left(\mathfrak{U}\left(V_{+}+b\right)\right)^{-}$. On the other hand, if $B \cdot E \Phi=0$ for some $B \in \pi\left(\mathfrak{U}\left(V_{+}+b\right)\right)^{-}$, one gets for a whole neighbourhood $\mathscr{N}$ of the origin in $\mathbb{R}^{4}$

$$
B \cdot E \cdot\left[\bigcup_{x \in \mathscr{N}} U(x) \cdot \mathfrak{F}^{\text {out }}(\mathcal{O}) \cdot U(x)^{-1} \cdot \Phi\right]=0
$$

because with our choice of $\mathcal{O}$ it follows that

$$
U(x) \cdot \mathscr{F}^{\text {out }}(\mathcal{O}) \cdot U(x)^{-1} C \pi\left(\mathfrak{U}\left(V_{+}+a\right)\right)^{-} \cap \pi\left(\mathfrak{U}\left(V_{+}+b\right)\right)^{\prime}
$$


if $x$ is small. Thus $B \cdot E=0$, and since $\pi\left(\mathfrak{U}\left(V_{+}+a\right)\right)^{-}$is a factor, $B=0$. Consequently $E \cdot \Phi$ is a separating vector for $\pi\left(\mathfrak{U}\left(V_{+}+b\right)\right)^{-}$, and this completes the proof of the statement. QED

The already mentioned, sharpened alternative holding for positive energy representations is now an immediate consequence of this lemma. (See the appendix of [2] for a similar argument.)

Proposition 2.3. Let $\pi_{1}, \pi_{2}$ be positive energy representations of $\mathfrak{A}$ whose restrictions to $\mathfrak{U}\left(V_{+}+a\right)$ are factorial. Then

- either $\pi_{1} \uparrow \mathfrak{U}\left(V_{+}+b\right) \simeq \pi_{2} \uparrow \mathfrak{U}\left(V_{+}+b\right)$ for $b \in \mathbb{R}^{4}$,

- or $\left.\pi_{1} \uparrow \mathfrak{U}\left(V_{+}+b\right)\right\rfloor \pi_{2} \uparrow \mathfrak{U}\left(V_{+}+b\right)$ for $b \in \mathbb{R}^{4}$,

where the symbol b denotes disjointness.

Proof. We must only show that the nondisjointness of $\pi_{1}$ and $\pi_{2}$ on $\mathfrak{U}\left(V_{+}+a\right)$ implies the equivalence of these representations on $\mathfrak{U}\left(V_{+}+b\right)$ for all $b \in \mathbb{R}^{4}$. Now any subrepresentation $\pi_{1}^{\prime}$ of $\pi_{1} \uparrow \mathfrak{U}\left(V_{+}+a\right)$ is of the form

$$
\pi_{1}^{\prime}(A)=\pi_{1}(A) \cdot E \quad \text { for } \quad A \in \mathfrak{U}\left(V_{+}+a\right)
$$

where $E \in \pi_{1}\left(\mathfrak{U}\left(V_{+}+a\right)\right)^{\prime}$ is a projection. From the previous lemma it then follows that $\pi_{1}^{\prime}$ and $\pi_{1}$ are unitarily equivalent on $\mathfrak{A}\left(V_{+}+b\right)$ for some $b$. A similar statement holds for $\pi_{2}$, hence if $\pi_{1} \uparrow \mathfrak{U}\left(V_{+}+a\right)$ and $\pi_{2} \uparrow \mathfrak{U}\left(V_{+}+a\right)$ have equivalent subrepresentations, then $\pi_{1} \uparrow \mathfrak{U}\left(V_{+}+b\right) \simeq \pi_{2} \uparrow \mathfrak{A}\left(V_{+}+b\right)$. This result extends to arbitrary $b$ because the representations $\pi_{1}$ and $\pi_{2}$ are invariant with respect to translations. QED

We can divide now the positive energy representations into charge-classes according to the following

Definition. Let $\pi$ be a positive energy representation which is factorial on $\mathfrak{U}\left(V_{+}\right)$. The charge-class $[\pi]$ of $\pi$ consists of the set of positive energy representations $\pi_{1}$ for which

$$
\pi_{1} \uparrow \mathfrak{U}\left(V_{+}\right) \simeq \pi \uparrow \mathfrak{U}\left(V_{+}\right)
$$

(It is an interesting question whether $\pi_{1}$ is then equivalent to $\pi$ also on the algebra $\mathfrak{U}\left(V_{-}\right)$. If this should not be the case, one must distinguish in the following analysis between the charge-classes $[\pi]_{V_{+}}$and $[\pi]_{V_{-}}$, respectively.)

It follows from the above proposition that the set of representations in a charge class $[\pi]$ is stable under taking direct sums and subrepresentations.

We will see that the charge-classes are a useful tool for the description of the localization properties of charged states. But before we can enter into that discussion, we must consider the question of how the charge-classes differ from each other. According to the above heuristic remarks it is plausible that representations of different charges belong to different charge-classes. The converse is, however, not true: we will indicate below that there exists a variety of such classes describing representations of a given electric charge which differ by some background radiation field.

Let us consider some irreducible positive energy representation $(\pi, \mathscr{H})$ of $\mathfrak{U}$ and study the algebras $\mathfrak{F}^{\text {in }}$ and $\mathfrak{F}^{\text {out }}$ of the asymptotic electromagnetic field on $\mathscr{H}$. 
We call the expectations of elements of $\mathfrak{F}^{\text {in }}$, respectively $\mathfrak{F}^{\text {out }}$, in a state the (incoming, respectively outgoing) radiation fields of that state. Now because of the presence of charged particles there will be in general substantial differences between the radiation fields of different states which may cause the corresponding subrepresentations of the asymptotic algebras $\mathfrak{F}^{\text {in }}$, respectively $\mathfrak{F}^{\text {out }}$, to be disjoint. But since $\pi$ is irreducible the radiation fields of the states in $\mathscr{H}$ can be transformed into each other by the action of local observables. Thus we may say that the differences between these fields are of a dynamical origin.

Let us compare next the radiation fields appearing in the charge class of $\pi$ : using Huygens' principle and Lemma 2.1 one can show that the algebra $\mathfrak{F}^{\text {out }}\left(V_{-}\right)^{-}$ is a factor, and assuming that the algebras $\mathfrak{F}^{\text {out }}\left(V_{-}\right)^{-}$and $\mathfrak{F}^{\text {in }}\left(V_{-}\right)^{-}$are isomorphic (which is clearly true if a scattering matrix exists) it follows that $\mathfrak{F}^{\text {in }}\left(V_{-}\right)^{-}$is a factor too. Therefore one can apply the arguments of Lemma 2.2 and show that the states in $\mathscr{H}$ induce equivalent representations of the algebra $\mathfrak{F}^{\text {in }}\left(V_{-}\right)^{-}$. Since $\mathfrak{F}^{\text {in }}\left(V_{-}\right)^{-}\left(\pi\left(\mathfrak{U}\left(V_{-}\right)\right)^{-}\right.$this result can then be extended to all states belonging to the charge-class of $\pi$. Thus the various representations of the incoming electromagnetic field appearing in the charge-classes of $\pi$ cannot be discriminated by measurements in $V_{-}$. (An analogous statement holds for the outgoing electromagnetic field.)

It is straightforward now to exhibit representations of the same electric charge as $\pi$ but belonging to different charge-classes: if $\omega$ is a vector-state with finite energy in the representation $\pi$, one can add to it (by means of creation operators) any number of incoming low-energy photons such that the resulting states $\omega_{n}$ have about the same energy as $\omega$. Hence each limit point $\omega^{\prime}$ of these states should induce a positive energy representation of $\mathfrak{A}[15]$ which, by construction, has the same electric charge as $\pi$. Now there exist sequences of states for which ${ }^{2}$

$$
\lim _{n} \|\left(\omega_{n}-\omega\right)\left\lceil\mathfrak{F}^{\mathrm{in}}\left(V_{-}+a\right) \|=2\right.
$$

for each $a \in \mathbb{R}^{4}$, and taking into account that $\mathscr{F}^{\text {in }}\left(V_{-}\right)^{-}$is a factor and that the free electromagnetic field commutes with itself at timelike distances, it follows from this relation that no limit point $\omega^{\prime}$ of the sequence $\omega_{n}$ is a normal state of $\mathfrak{F}^{\text {in }}\left(V_{-}\right)^{-}$. Hence the states $\omega$ and $\omega^{\prime}$ do not belong to the same charge-class although they do have the same electric charge.

In view of the above remarks it is clear that the states $\omega$ and $\omega^{\prime}$ differ by radiation fields which cannot be transformed into each other by the action of local observables. In order to stress this point we say that these states have different background radiation fields. Thus the charge-classes are not only labeled by the electric charge but also by such background fields.

\section{States of Interest}

Up to this point we have considered in our analysis the whole set of physical states in quantum electrodynamics. Now we want to restrict our attention to the subset of states which do not have any background radiation field. The charge-classes

2 This statement can easily be verified because it refers only to the free electromagnetic field 
generated by this restricted set of states are expected to be uniquely characterized by their electric charge, and we will incorporate this idea in the subsequent selection criterion. Moreover, since the vacuum does not have a background field, we can take it as a reference state, and we will identify all other states of interest by their good localization properties with respect to the vacuum. The formal conditions characterizing these states are the following ones.

Criterion. Let $\omega$ be a state inducing a representation $(\pi, \mathscr{H})$ of $\mathfrak{U}$. $\omega$ is called an infrared minimal state (and $\pi$ an infrared minimal representation) if the following conditions are satisfied:

i) $\pi$ is a positive energy representation.

ii) $\pi \circ \alpha_{\Lambda} \in[\pi]$ for all Lorentz transformations $\Lambda$.

iii) For each open, pointed spacelike $\operatorname{cone}^{3} \mathscr{S} \subset \mathbb{R}^{4}$ there exists a representation $\pi_{\mathscr{S}} \in[\pi]$ such that

$$
\pi_{\mathscr{S}} \uparrow \mathfrak{U}\left(\mathscr{S}^{\prime}\right) \simeq \pi_{0} \uparrow \mathfrak{U}\left(\mathscr{S}^{\prime}\right)
$$

where $\pi_{0}$ is the vacuum representation.

It is obvious that the vacuum induces an infrared minimal representation in the sense of this selection criterion, and we will now provide some heuristic arguments to the effect that the criterion admits also states carrying an electric charge. The first condition needs no explanation, so let us consider the second one. This condition says that the states in the representations $\pi \circ \alpha_{A}$ and $\pi$ cannot be distinguished by a classical observable which can be determined in a lightcone. Since the electric charge is Lorentz invariant, this requirement should not exclude charged states, but we expect that it rules out states with a background radiation field. For the infrared minimal representations it is noteworthy that for each $\pi_{1} \in[\pi]$ one has $\pi_{1} \circ \alpha_{A} \in\left[\pi \circ \alpha_{A}\right]=[\pi]$ because Lorentz transformations map lightcones onto lightcones. Thus stability of the charge-classes under Lorentz transformations seems to be the appropriate substitute for the general lack of invariance of the individual representations [4].

The third condition of the criterion expresses the assumption that one can prescribe the shape of the asymptotic electromagnetic field of the infrared minimal states of a given charge. Of course, one must choose suitable data in order not to come into conflict with the first two conditions of the criterion. But taking classical electrodynamics as a guide, (which is legitimate in this context because all that matters is the behaviour of the field at infinity, which is essentially classical) it seems to be plausible that one can find states of a given electric charge which have finite energy and whose electromagnetic field at time $t$ is confined (in the sense of Cauchy-data) to some cone $\mathscr{S} \subset \mathbb{R}^{3}$ without giving rise to a background radiation field ${ }^{4}$. Because of locality, it would then not be possible to distinguish such a state from states in the vacuum representation by measurements in the causal complement $\mathscr{S}^{\prime}$ of the region $(t, \mathscr{S})$, and this is the content of relation (3.1).

3 It suffices to consider cones $\mathscr{S}$ of the form $\mathscr{S}=a+\bigcup_{\lambda>0} \lambda \cdot \mathcal{O}$, where $a \in \mathbb{R}^{4}$ and $\mathcal{O}$ is an open double cone whose closure lies in the spacelike complement of the origin in $\mathbb{R}^{4}$

4 In classical electrodynamics such background fields are connected with Cauchy-data which decrease more slowly than the Coulomb field, i.e. than $|\mathbf{x}|^{-2}$ 
In a field-theoretic setting it should be possible to construct the infrared minimal states with the help of Coulomb-type field operators $\psi_{c}$, which are formally related to the Fermi-field $\psi$ and the vector-potential $A_{\mu}$ in the GuptaBleuler formalism according to

$$
\psi_{c}(x)=\psi(x) \cdot \exp \left(i e \int d^{4} y c^{\mu}(x-y) A_{\mu}(y)\right) .
$$

The functions $c_{\mu}$ have to satisfy the equation

$$
\partial^{\mu} c_{\mu}(x)=\delta(x)
$$

because $\psi_{c}$ must be invariant under local gauge-transformations. Moreover, in order not to give rise to any background field, $c_{\mu}$ should decrease in all directions of $\mathbb{R}^{4}$ at least like $|x|^{-3}$; this is the optimal behaviour which is compatible with this equation. The set of these functions $c_{\mu}$ is stable under Lorentz transformations, and for any open spacelike cone $\mathscr{S}$ containing the origin of Minkowski space there exist functions $c_{\mu}$ having support in $\mathscr{S}$. Therefore one may expect that the appropriately regularized field-operators $\psi_{c}$ generate charged states from the vacuum which belong to the set of infrared minimal states. Yet since it is not quite clear whether one can give a rigorous meaning to the fields $\psi_{c}$ and since the concept of a charge class does not fit very well into a fieldtheoretic setting, we will use here the more intrinsic characterization of infrared minimal states given in the criterion.

We remark, as an aside, that our selection criterion leads also to a complete characterization of the physical state space in massive particle theories. It is evident that in such a theory the energy of a state can be determined in each lightcone of Minkowski space, similar to the electric charge in quantum electrodynamics. Therefore, the time translation operators $U(t)$ are elements of the algebras $\pi\left(\mathfrak{U}\left(V_{ \pm}\right)\right)^{-}$, and consequently $\pi\left(\mathfrak{H}\left(V_{ \pm}\right)\right)^{-}=\pi(\mathfrak{R})^{-}$. (For a formal argument see [16].) This implies that the notions of charge class and sector are synonymous in massive theories. Thus the second part of the criterion amounts to the condition that each representation $\pi$ of interest has to be invariant under Lorentz transformations, and the third part means that $\pi$ must be equivalent to the vacuum representation on the algebras $\mathfrak{2}\left(\mathscr{S}^{\prime}\right)$ for each spacelike cone $\mathscr{S}$. It has recently been shown (without making any assumptions on charge-carrying fields) that all particle states in a massive, Poincaré covariant theory fulfill these conditions [17].

We believe that the infrared minimal states constitute the smallest set of states which is stable under Poincare transformations and which contains charged states with optimal localization properties relative to the vacuum. Therefore we propose to consider this set as the physical state space of quantum electrodynamics. But we recall that this choice of states is merely a convention, which we adopt here because on the one hand these states exhibit the basic features of quantum electrodynamics, and on the other hand they are a convenient starting point for a general structural analysis.

In conclusion let us mention two other possibilities of choosing a physical state space which have been discussed in the literature. The first one amounts to picking from each charge class a particular representation and then taking their direct sum. This is what one does in a field-theoretic setting if one generates the physical 
states from the vacuum by means of a fixed Coulomb-field $\psi_{c}$. If the localization properties of the field $\psi_{c}$ (i.e. the functions $c_{\mu}$ ) are held fixed, it is, however, difficult to exploit locality in the analysis of the physical state space. Therefore we consider here states with arbitrary localization properties. This forces us to deal with many inequivalent representations, but if these representations belong to a fixed chargeclass, we know how they are related, and this is an important additional piece of information embodied in our setting.

The second alternative consists in selecting states which have a specific background field. That such a choice might have its virtues has been pointed out by Kraus et al. [18]. The basic idea in this approach is to add to the vacuum state a background field which is sufficiently strong such that the radiation fields which are produced in collisions can be regarded as small perturbations of it. All states in such an infra-vacuum representation $\bar{\pi}_{0}$ of $\mathfrak{U}$ are expected to induce quasiequivalent representations of the algebra generated by the incoming or outgoing electromagnetic field, respectively. So, in a sense, the asymptotic radiation fields are, in these representations, decoupled from the momenta of the charged particles, and one may hope that this simplifies their description in collision processes [18].

Charged states could be obtained in this setting by applying the Coulomb-field operators $\psi_{c}$ to the vector representing the infra-vacuum. We conjecture that the corresponding representations $\bar{\pi}$ are equivalent to the infra-vacuum representation $\bar{\pi}_{0}$ on the algebras $\mathfrak{U}\left(\mathscr{S}^{\prime}\right)$ for arbitrary spacelike cones $\mathscr{S}$,

$$
\bar{\pi} \uparrow \mathfrak{A}\left(\mathscr{S}^{\prime}\right) \simeq \bar{\pi}_{0} \uparrow \mathfrak{U}\left(\mathscr{S}^{\prime}\right),
$$

because the background field should make it impossible to discriminate the asymptotic flux-distributions of these representations. (It is only the total electric flux on which the background field has no effects.) Hence with regard to the localization properties of the representations $\bar{\pi}$, one would be in a situation similar to that discussed in [17] for massive theories, so from this point of view this scheme also looks attractive.

Unfortunately, one does not yet have sufficient control on the properties of the infra-vacuum representations $\bar{\pi}_{0}$ to take them as a starting point for an analysis of the infrared problems in quantum electrodynamics. Moreover, it is to be expected that a discussion of the asymptotics of the charged particles (the infra-particle problem [19]) will be more difficult in these representations than in the infrared minimal representations, because one has to take into account the response of the charged particles to the background field. In view of these difficulties we have chosen to restrict our attention to the infrared minimal states, because they describe the most transparent idealizations of actual experimental situations.

\section{Scattering Observables}

In this section we want to show how one can extract from the defining properties of the infrared minimal states information about the structure of the observables at asymptotic times. This question is intimately related to the infrared-problem, and we want to clarify here some of the basic facts concerning this matter. 
Our main result in this context is that asymptotic electromagnetic fields $F^{\text {in }}$ and $F^{\text {out }}$ can be defined as LSZ-type of limits of suitable local observables in all infrared minimal representations. For the proof of this assertion it is essential that there exist representations in a given charge-class which are localized in spacelike cones. This fact enables us to extend the results of [12] (where the existence of asymptotic electromagnetic fields has been established for the vacuum representation $\pi_{0}$ ) to all representations of interest.

Let $F=F^{*} \in \mathfrak{A}$ be any local observable such that $\pi_{0}(F)$ connects the vacuum with a single photon state. In addition we assume that the function $x \rightarrow \alpha_{x}(F) \equiv F(x)$ is smooth in the norm-topology on $\mathfrak{U}$ and that its Fourier-transform is sufficiently regular at the origin in a sense made precise in $[12]^{5}$. Then we define for each $t \in \mathbb{R}$

$$
F_{t}(f)=-2 t \cdot \int d \omega f(\mathbf{n}) \partial_{0} F(t, t \cdot \mathbf{n})
$$

where $d \omega=d \omega(\mathbf{n})$ is the normalized, invariant measure on the unit sphere $S_{2}, f(\mathbf{n})$ is a real, smooth function on $S_{2}$ and $\partial_{0}$ denotes differentiation with respect to the time-translations. The observables $F_{t}(f)$ are designed to approximate the asymptotic electromagnetic fields in the limit of large $t$. In order to improve the convergence of these sequences it is necessary to take suitable time-averages of $F_{t}(f)$, as for example $(|t|>1)$

$$
\bar{F}_{t}(f)=\frac{1}{\ln |t|} \cdot \int_{t}^{t+\ln |t|} d t^{\prime} F_{t^{\prime}}(f) .
$$

It then follows from [12] that these sequences converge for $t \rightarrow \pm \infty$ (in the sense of strong resolvent convergence) in the vacuum representation to asymptotic fields which have all properties expected from a smoothed-out, free massless field. Actually, this has only been demonstrated for the case $f=1$, but the argument in [12] is valid for arbitrary smooth functions $f$. Amongst the properties of the asymptotic fields which are of relevance here we mention that

$$
s-\lim _{t \rightarrow \pm \infty} \pi_{0}\left(\bar{F}_{t}(f)\right) \Omega=E^{(1)} \cdot f\left(\frac{\mathbf{P}}{|\mathbf{P}|}\right) \cdot \pi_{0}(F) \Omega,
$$

where $E^{(1)}$ is the projection onto the single-photon space $\mathscr{H}_{0}^{(1)}$ and $\mathbf{P}$ is the momentum operator in the vacuum representation. Furthermore,

$$
s-\lim _{t \rightarrow \pm \infty} \pi_{0}\left(e^{i \bar{F}_{t}(f)} \cdot e^{i \bar{F}_{t}^{\prime}\left(f^{\prime}\right)} \cdot e^{-i \bar{F}_{t}(f)-i \bar{F}_{t}^{\prime}\left(f^{\prime}\right)}\right)=\zeta \cdot 1
$$

where $\zeta$ is a phase-factor. Hence the exponentials of the asymptotic fields have the familiar Weyl-commutation relations.

In order to establish the existence of these limits for all infrared minimal representations $\pi$ we proceed as follows : first we pick some pointed, spacelike cone $\mathscr{S}$ with apex at the origin of Minkowski space and a representation $\pi_{\mathscr{S}} \in[\pi]$ which is equivalent to $\pi_{0}$ on $\mathfrak{A}\left(\mathscr{S}^{\prime}\right)$. Then we exploit the simple geometrical fact that there exists some open set $\boldsymbol{\Sigma} \subset S_{2}$ such that $\mathscr{O}_{1}+(t, t \cdot \Sigma) \subset \mathscr{S}^{\prime}$ for each bounded region $\mathcal{O}_{1} \subset V_{-}$and sufficiently large positive $t$. Hence if we choose in the defining relation

5 Since the representations of interest are locally normal with respect to $\pi_{0}$ these two conditions can be met by extending the local algebras, if necessary 
(4.1) any function $f$ with support in $\boldsymbol{\Sigma}$ and an observable $F \in \mathfrak{U}\left(\mathcal{O}_{1}\right)$ it follows that $\bar{F}_{t}(f) \in \mathfrak{U}\left(\mathscr{S}^{\prime}\right)$ for large $t$. Because of the equivalence of $\pi_{\mathscr{S}}$ and $\pi_{0}$ on $\mathfrak{U}\left(\mathscr{S}^{\prime}\right)$ it is then clear that the strong limit

$$
S-\lim _{t \rightarrow \infty} \pi_{\mathscr{S}}\left(e^{i \bar{F}_{t}(f)}\right)
$$

exists. Moreover, since the representation $\pi_{\mathscr{S}}$ is invariant under translations this result extends to observables $F$ which are localized in arbitrary bounded regions $\mathcal{O} \subset \mathbb{R}^{4}$. By the same token one finds that for functions $f, f^{\prime}$ with support in $\Sigma$ the commutation relations (4.4) hold in the representation $\pi_{\mathscr{S}}$ with the same $\zeta$ as in the vacuum representation. Hence we have established the existence of outgoing electromagnetic fields in the representation $\pi_{\mathscr{S}}$, whose momentum transfer is, however, restricted to the set $\left\{\mathbf{p}: \frac{\mathbf{p}}{|\mathbf{p}|} \in \pm \Sigma\right\}$, as can be seen from relation (4.3). Now we exploit relation (2.2) relating the representations within a charge-class: if $F \in \mathfrak{U}(\mathcal{O})$ we have by construction $e^{i \bar{F}_{t}(f)} \in \mathfrak{U}\left(\mathcal{O}+V_{+}\right)$for positive $t$, and since $\pi_{\mathscr{S}} \in[\pi]$ it follows from relations (2.2) and (4.5) that the limit

$$
S-\lim _{t \rightarrow \infty} \pi\left(e^{i \bar{F}_{t}(f)}\right)
$$

exists if $\operatorname{supp} f \subset \boldsymbol{\Sigma}$. This argument holds for each choice of the cone $\mathscr{S}$, and it is then clear that the limit (4.6) exists for smooth functions $f$ which have support in sufficiently small regions generating some covering of $S_{2}$.

After having established the existence of asymptotic fields $F^{\text {out }}(f)$ for these special functions we will now extend this result to $f=1$; the corresponding fields $F^{\text {out }}(1)=F^{\text {out }}$ can then be used to generate the local algebras of the outgoing electromagnetic field. Since it is not clear to us whether the limit (4.6) exists if $f=1$, we define $F^{\text {out }}$ by exploiting the linearity of $F^{\text {out }}(f)$ in $f$ and the $c$-number commutation relations of free fields. In order to illustrate this idea let us assume for a moment that $F^{\text {out }}$ exists. Then, if $\sum_{1}^{n} f_{i}=1$ is a partition of unity for a covering
of $S_{2}$, we could write

$$
e^{i F^{\text {out }}}=\zeta^{\prime} \cdot e^{i F^{\text {out }}\left(f_{1}\right)} \ldots e^{i F^{\text {out }}\left(f_{n}\right)},
$$

where $\zeta^{\prime}$ is some phase-factor. Now if the regions in the covering of $S_{2}$ are sufficiently small then each operator on the right hand side of (4.7) exists, and we can interpret this relation as the definition of $e^{i F^{\text {out }}}$. We must only verify that this definition is consistent, i.e. that it does not depend on the choice of the partition of unity.

According to these remarks we define $e^{i F^{\text {out }}}$ as the limit

$$
s-\lim _{t \rightarrow \infty} \pi\left(\zeta^{\prime} \cdot e^{i \bar{F}_{t}\left(f_{1}\right)} \ldots e^{i \bar{F}_{t}\left(f_{n}\right)}\right),
$$

where $\zeta^{\prime}$ is given by

$$
\zeta^{\prime}=\lim _{t \rightarrow \infty} \frac{\left|\omega_{0}\left(e^{i \bar{F}_{t}\left(f_{1}\right)} \ldots e^{i \bar{F}_{t}\left(f_{n}\right)}\right)\right|}{\omega_{0}\left(e^{i \bar{F}_{t}\left(f_{1}\right)} \ldots e^{i \bar{F}_{t}\left(f_{n}\right)}\right)}
$$

and $\omega_{0}$ is the vacuum state. Using the commutation relations (4.4) for functions $f, f^{\prime}$ which both have support in a sufficiently small region $\boldsymbol{\Sigma} \subset S_{2}$ as well as the fact 
that because of locality

$$
\left[e^{i \bar{F}_{t}(f)}, e^{i \bar{F}_{t}\left(f^{\prime}\right)}\right]=0
$$

if $t$ is large and $f, f^{\prime}$ have disjoint supports, it is easy to prove that the limit (4.8) indeed does not depend on the chosen partition $\sum_{1}^{n} f_{i}=1$.

We now define a net $\mathcal{O} \rightarrow \mathfrak{F}^{\text {out }}(\mathcal{O})^{6}$ of von Neumann algebras in $\pi(\mathfrak{A})^{-}$by setting for each double cone $\mathcal{O}_{1}$ and sufficiently regular observables $F$

$$
\mathfrak{F}^{\text {out }}\left(\mathcal{O}_{1}\right)=\left\{e^{i F^{\text {out }}}: F=F^{*} \in \mathfrak{A}\left(\mathcal{O}_{1}\right)\right\}^{\prime \prime} \text {. }
$$

The algebras for arbitrary regions $\mathcal{O}$ are then obtained by addivity. Applying the arguments in [12] it is straightforward to verify that the net $\mathcal{O} \rightarrow \mathfrak{F}^{\text {out }}(\mathcal{O})$ is local and covariant with respect to the action of the translations $U(x)$. Moreover, if OC $C V_{+}+a$ we get by construction

$$
\mathfrak{F}^{\text {out }}(\mathcal{O}) \subset \pi\left(\mathfrak{U}\left(V_{+}+a\right)\right)^{-},
$$

and if $\mathscr{O} \subset V_{-}+b$ it follows from locality that

$$
\mathfrak{F}^{\text {out }}(\mathcal{O}) \subset \pi\left(\mathfrak{U}\left(V_{+}+b\right)\right)^{\prime}
$$

which is Huygens' principle. (These are the structural relations anticipated in Sect. 2.) Hence we get

Theorem 4.1. Let $(\pi, \mathscr{H})$ be an infrared minimal representation. Then there exists a local, covariant net $\mathcal{O} \rightarrow \mathfrak{F}^{\text {out }}(\mathcal{O})$ on $\mathscr{H}$ which is generated by the outgoing (free) electromagnetic field and satisfies

i) $\mathfrak{F}^{\text {out }}(\mathcal{O}) \subset \pi\left(\mathfrak{U}\left(V_{+}+a\right)\right)^{-}$if $\mathcal{O} \subset V_{+}+a$.

ii) $\mathfrak{F}^{\text {out }}(\mathcal{O}) \subset \pi\left(\mathfrak{A}\left(V_{+}+b\right)\right)^{\prime}$ if $\mathcal{O} \subset V_{-}+b$.

(A similar statement holds for the incoming field.)

Let us now turn to a discussion of the asymptotic observables which are sensitive to the massive particles. According to the general ideas of Araki and Haag [20], it should be possible to obtain these observables as time-limits of suitable averages of almost local observables $C \in \mathfrak{U}$, such as

$$
t^{3} \cdot \int d^{3} v h(\mathbf{v}) \cdot C(t, t \cdot \mathbf{v})
$$

where $h$ is a function which has support in the region $|\mathbf{v}|<1$, and $C$ annihilates the vacuum state, i.e. $\omega_{0}\left(\mathrm{C}^{*} \mathrm{C}\right)=0$. Unfortunately, we are not aware of a rigorous argument establishing the existence of any reasonable limit of these sequences in quantum electrodynamics, and it is conceivable that one has to proceed in a rather indirect way in order to gain control on the limits (as in the case of the electromagnetic field).

One may, however, expect from the asymptotic localization properties of the observables (4.14), as well as from the fact that outgoing massive particles will eventually enter each cone $V_{+}+a$, that for any reasonable definition of the algebra

6 An unambiguous, but more clumsy notation would be $\mathfrak{F}_{\pi}^{\text {out }}(\mathcal{O})$ 
$\mathfrak{M}^{\text {out }}$ of all massive observables at positive asymptotic times in a representation $\pi$ one has

$$
\mathfrak{M}^{\text {out }} \subset \bigcap_{a} \pi\left(\mathfrak{U}\left(V_{+}+a\right)\right)^{-} .
$$

This relation and Huygens' principle imply that $\mathfrak{M}^{\text {out }} \subset \mathfrak{F}^{\text {out', where }}$ $\mathfrak{F}^{\text {out }}=\bigcup_{\mathbb{O}}^{C^{\star}} \mathfrak{F}^{\text {out }}(\mathcal{O})$. Hence we obtain for the algebra $\mathfrak{A}^{\text {out }}=\mathfrak{F}^{\text {out }} \cup \mathfrak{M}^{\text {out }}$ of all outgoing observables the inclusion

$$
\mathfrak{H}^{\text {out }} \subset \mathfrak{F}^{\text {out }-\vee} \vee \mathfrak{F}^{\text {out' }}
$$

It follows from this relation that the algebra $\mathfrak{A}^{\text {out }}$ is reducible if $\mathfrak{F}^{\text {out }-}$ has a center (which is to be expected if $\pi$ is an infrared-minimal representation [22]). In that case the asymptotic observables $\mathfrak{Q}^{\text {out }}$ do not yield a complete characterization of the physical states, and the same is true for $\mathfrak{A}^{\text {in }}$. Hence a scattering matrix cannot uniquely be fixed in quantum electrodynamics by the condition that it induces a mapping of the outgoing observables onto their respective incoming counterparts. These difficulties are related to the well-known fact that there does not exist an asymptotic position operator for the charged particles in quantum electrodynamics.

There should, however, exist for each $a \in \mathbb{R}^{4}$ sufficiently many observables in $\mathfrak{U}\left(V_{+}+a\right)$ in order to completely determine the properties of the outgoing massive particles in the states of interest. In fact, it seems to be a reasonable assumption that all observables in an irreducible infrared-minimal representation $\pi$, which are commensurable with $\pi\left(\mathfrak{U}\left(V_{+}+a\right)\right)$, are contained in $\mathfrak{F}^{\text {out }}\left(V_{-}+a\right)^{-7}$. This property, which may be regarded as a weak form of asymptotic completeness, guarantees that the algebra $\pi\left(\mathfrak{U}\left(V_{+}+a\right)\right)^{-}$contains the full information about the outgoing massive particles. For later reference we give the following

Definition. Let $\pi$ be an infrared minimal representation. $\pi$ has the property of weak asymptotic completeness if

$$
\pi\left(\mathfrak{U}\left(V_{+}\right)\right)^{\prime}=\mathfrak{F}^{\text {out }}\left(V_{-}\right)^{-} \text {and } \pi\left(\mathfrak{U}\left(V_{-}\right)\right)^{\prime}=\mathfrak{F}^{\text {in }}\left(V_{+}\right)^{-}
$$

In order to substantiate this concept we will now show that weak asymptotic completeness holds for the vacuum representation $\left(\pi_{0}, \mathscr{H}_{0}\right)$. In the proof we will make the assumption that in quantum electrodynamics there exist only massive charged particles and that in collisions of low-energy photons (with total energy below the pair creation threshold) no infrared-clouds of photons are produced. More precisely: all vectors in $\mathscr{H}_{0}$ with total mass below some sufficiently small threshold mass $\mu>0$ should belong to the Fock spaces of incoming and outgoing photons respectively, i.e.

$$
E_{\mu} \mathscr{H}_{0} \subset\left[\mathscr{F}^{\text {in }} \Omega\right] \cap\left[\mathscr{F}^{\text {out }} \Omega\right]
$$

where $E_{\mu}$ denotes the projection onto the vectors in $\mathscr{H}_{0}$ with total mass smaller than $\mu$. From this assumption we get

7 The inclusion $\mathfrak{F}^{\text {out }}\left(V_{-}+a\right) \subset \pi\left(\mathfrak{A}\left(V_{+}+a\right)\right)^{\prime}$ always holds because of Huygens' principle 
Proposition 4.2. $\pi_{0}$ has the property of weak asymptotic completeness.

Proof. It has been shown in [12] that the subrepresentation of the outgoing electromagnetic field on $\left[\mathfrak{F}^{\text {out }} \Omega\right]$ is equivalent to the Fock-representation. Therefore we can apply the arguments in [21] establishing the timelike duality relation

$$
\left(\mathfrak{F}^{\text {out }}\left(V_{+}\right) \cdot E^{\text {out }}\right)^{\prime}=\mathfrak{F}^{\text {out }}\left(V_{-}\right)^{-} \cdot E^{\text {out }},
$$

where $E^{\text {out }} \in \mathfrak{F}^{\text {out' }}$ is the projection onto [ $\mathfrak{F}^{\text {out }} \Omega$ ]. Now if $X \in \pi\left(\mathfrak{H}\left(V_{+}\right)\right)^{\prime} \subset \mathfrak{F}^{\text {out }}\left(V_{+}\right)^{\prime}$ then $E^{\text {out }} \cdot X \cdot E^{\text {out }} \in\left(\mathscr{F}^{\text {out }}\left(V_{+}\right) \cdot E^{\text {out }}\right)^{\prime}$ and consequently there exists some $X^{\text {out }} \in \mathfrak{F}^{\text {out }}\left(V_{-}\right)^{-}$such that $E^{\text {out }} \cdot X \cdot E^{\text {out }}=X^{\text {out }} \cdot E^{\text {out }}$.

In order to see that $X=X^{\text {out }}$ we proceed as follows : according to relation (4.17) the vector

$$
\left(X-X^{\text {out }}\right) \Omega=\left(X-E^{\text {out }} \cdot X^{\text {out }}\right) \Omega=\left(1-E^{\text {out }}\right) \cdot X \Omega
$$

has a minimal mass which is larger than $\mu$, and the same holds for $\left(X^{*}-X^{\text {out } *}\right) \Omega$. Therefore the Fourier transform of

$$
t \rightarrow K(t)=\left(\Omega,\left[\pi_{0}\left(\alpha_{t}(A)\right),\left(X-X^{\mathrm{out}}\right)\right] \Omega\right)
$$

(where $\alpha_{t}$ are the time-translations) vanishes in $(-\mu, \mu)$. If in addition $A \in \mathfrak{U}\left(V_{+}\right)$ then $K(t)$ vanishes for $t>0$ which implies that its Fourier-transform $\tilde{K}(\omega)$ is the boundary value of an analytic function, hence $\tilde{K}(\omega)$ vanishes everywhere. It then follows that $\left(X-X^{\text {out }}\right) \Omega=0$, and since the vacuum is separating for $\pi_{0}\left(\mathfrak{A}\left(V_{+}\right)\right)^{\prime}$ we arrive at $X=X^{\text {out }}$. This shows that $\pi_{0}\left(\mathfrak{U}\left(V_{+}\right)\right)^{\prime}=\mathfrak{F}^{\text {out }}\left(V_{-}\right)^{-}$, and by an analogous argument we get $\pi_{0}\left(\mathfrak{U}\left(V_{-}\right)\right)^{\prime}=\mathfrak{F}^{\text {in }}\left(V_{+}\right)^{-}$. QED.

Because of this result we expect that weak asymptotic completeness holds for all irreducible infrared-minimal representations in quantum electrodynamics, and we will later make use of this hypothesis. We conclude the present section with some remarks on the infra-particle problem in quantum electrodynamics [19]. A detailed analysis of this subject can be found in [22] and we want to refine some of these results in the present setting.

First we recall that in each factorial positive energy representation $\pi$ of a local net there exist unique translation operators $U(x)$ whose generators have joint spectrum with a Lorentz invariant lower boundary [7]. Moreover, if $\pi$ is an infrared minimal representation one has $U(x) \mathfrak{F}^{\text {out }} U(x)^{-1}=\mathfrak{F}^{\text {out }}$, and consequently there exist translation operators $U_{\mathfrak{F}}^{\text {out }}(x) \in \mathfrak{F}^{\text {out }-}$ inducing the same action on $\mathfrak{F}^{\text {out }}$ as $U(x)[10]$. Using the fact that $\mathfrak{F}^{\text {out }}$ is generated by a free massless field it is then easy to prove

Proposition 4.3. There exists a unique decomposition

$$
U(x)=U_{\widetilde{F}}^{\text {out }}(x) \cdot U_{\mathfrak{M}}^{\text {out }}(x),
$$

where $x \rightarrow U_{\widetilde{F}}^{\text {out }}(x) \in \mathfrak{F}^{\text {out }-}$ and $x \rightarrow U_{\mathfrak{M}}^{\text {out }}(x) \in \mathfrak{F}^{\text {out }}$ are continuous, unitary representations of the translations. The spectrum of the generators of $U_{\mathscr{F}}^{\text {out }}(x)$ is equal to $\bar{V}_{+}$ in each subrepresentation of $\mathfrak{F}^{\text {out }}$, and the spectra of $U_{\mathfrak{M}}^{\text {out }}(x)$ and $U(x)$ have the same Lorentz invariant lower boundary.

(For an explicit construction of $U_{\widetilde{F}}^{\text {out }}(x)$ see [22].) 
Let us focus now on representations $(\pi, \mathscr{H})$, where states consisting of a single charged particle and any number of photons appear. Thinking of quantum electrodynamics it is reasonable to assume that the states in $\mathscr{H}$ which are attached to an arbitrarily small neighbourhood of the lower boundary $H_{m}=\left\{p: p^{2}=m^{2}, p_{0}>0\right\}$ of $\operatorname{sp} \mathscr{U}$ contain this particle and that by adding sufficiently many photons to these states one obtains all states in $\mathscr{H}$ with masses below some threshold mass $M>m$. This means that

$$
E_{M} \mathscr{H} \subset\left[\mathscr{F}^{\text {in }} \cdot E_{m+\delta} \mathscr{H}\right] \cap\left[\mathscr{F}^{\text {out }} \cdot E_{m+\delta} \mathscr{H}\right]
$$

for any $\delta>0$, where $E_{\lambda}$ denotes the projection in $\mathscr{H}$ onto the states with total mass smaller than $\lambda$. It is then an immediate consequence that the lower boundary of $\operatorname{sp} \mathscr{U}_{\mathfrak{M}}^{\text {out }}$ is isolated from the rest of the spectrum.

Proposition 4.4. Let $U_{\mathfrak{M}}^{\text {out }}(x)$ be the translations defined in the preceding proposition. Then

$$
H_{m} \subset \operatorname{sp} \mathscr{U}_{\mathfrak{M}}^{\text {out }} \subset H_{m} \cup\left\{p: p^{2} \geqq M^{2}, p_{0}>0\right\} .
$$

(This property of $U_{\mathfrak{M}}^{\text {out }}(x)$ has been noticed first in [22].) These results show that energy and momentum of the outgoing photons and charged particles, respectively, are commensurable observables. Moreover, the charged particles have a definite mass $m$. It is also noteworthy that

$$
U_{\mathfrak{M}}^{\text {out }}(x) \in \bigcap_{a} \pi\left(\mathfrak{U}\left(V_{+}+a\right)\right)^{-}
$$

if weak asymptotic completeness holds for $\pi$, hence the charged particles have all properties expected on physical grounds.

The famous infra-particle problem in quantum electrodynamics consists now in the fact that every state on $\mathfrak{A}$ describing particles which carry an electric charge also contains photons. This implies that these particles do not correspond to a discrete eigenvalue of the full mass-operator $P^{2}$, they are infra-particles [19]. The fact that there is no way of removing all photons from these states can be expressed using the concept of charge-classes: there exists no representation in the charge-class of $\pi$ where the mass-operator has a discrete eigenvalue. This suggests the following general characterization of representations including infra-particles.

Criterion. An infrared-minimal representation $\pi$ includes an infraparticle of mass $m$ if weight.

i) $\operatorname{sp} \mathscr{U}_{\mathfrak{M}}^{\text {out }}$ (respectively $\operatorname{sp} \mathscr{U}_{\mathfrak{M}}^{\text {in }}$ ) contains the hyperboloid $H_{m}$ with discrete

ii) the translations $\mathscr{U}_{1}$ have continuous spectrum about $H_{m}$ in each representation $\pi_{1} \in[\pi]$.

That electrically charged particles appear as infra-particles in a field-theoretic setting can be understood from the following heuristic argument: each charged particle with mass $m$ and momentum about $\mathbf{p}$ produces an electric fluxdistribution, which at sufficiently large distances from the particle (such that one can apply classical physics) has the form

$$
\varphi_{\mathbf{p}}(\mathbf{n})=\frac{e m^{2}}{4 \pi} \cdot \frac{\left(\mathbf{p}^{2}+m^{2}\right)^{1 / 2}}{\left((\mathbf{p n})^{2}+m^{2}\right)^{3 / 2}}
$$


where $\mathbf{n}$ denotes the direction in which one looks. Thus charged single particle states with different momenta produce different asymptotic flux-distributions. On the other hand it follows from locality that all states in a factorial representation $\pi$ of $\mathfrak{U}$ have the same asymptotic flux-distribution $\varphi_{\pi}$. Therefore each state describing a charged particle with momentum about $\mathbf{q}$ must (for almost all $\mathbf{q}$ ) also include some radiation field which adds to the asymptotic flux-distribution $\varphi_{\mathbf{q}}$ of the bare particle to give the net flux-distribution $\varphi_{\pi}$ fixed by the representation. Since the radiation field carries some (though arbitrarily small) energy, it is clear that no such state can have a precise mass.

From the same argument it follows also that if one changes the momentum of a charged particle by a local operation from $\mathbf{q}$ to $\mathbf{p}$ say, one inevitably produces also some radiation field which induces the asymptotic flux-distribution $\varphi_{\mathbf{q}}(\mathbf{n})-\varphi_{\mathbf{p}}(\mathbf{n})$. This is exactly what one expects from classical electrodynamics.

Finally we remark that one can determine the asymptotic momentum of a charged particle by measuring the electric flux emanating from it. One must only wait long enough, until all hard photons have separated from the particle such that its momentum stays essentially constant. Then the electric flux-distribution should have the form (4.20) in a large region about the particle (provided no background radiation field is present), and from this one can read off the momentum of the particle. The fact that one can determine the asymptotic momentum of a charged particle far away from its actual localization region, indicates that this observable should be commensurable with all other asymptotic observables which are sensitive to this particle. Hence the asymptotic momentum should be an element of the center of $\mathfrak{M}^{\text {out }}$ (respectively $\mathfrak{M}^{\text {in }}$ ). This is consistent with the conjecture made in [22] that in a situation where all massive particles carry an electric charge, $\mathfrak{M}^{\text {out }}$ and $\mathfrak{M}^{\text {in }}$ should be Abelian algebras generated by the asymptotic particle momenta.

We emphasize that in these qualitative arguments only Gauss' law, locality and the coherence of states within a sector have been used. This indicates that the above features of electrically charged particles are independent of the detailed dynamical structure of quantum electrodynamics, so that one may hope to establish them rigorously in the present general setting.

\section{Radiation Fields}

After this survey of the structure of the scattering observables in quantum electrodynamics, we are now in a position to determine the relation between the representations within a charge-class. We shall see that these representations describe states which differ only by some radiation field, so in particular they carry the same electric charge. This shows that the heuristic ideas which led us to the concept of charge-classes are consistent.

Now let $(\pi, \mathscr{H})$ be any positive energy representation. If $\left(\pi_{1}, \mathscr{H}_{1}\right)$ is an element of the charge-class of $\pi$ there exists some isometry $V$ from $\mathscr{H}_{1}$ onto $\mathscr{H}$ such that

$$
V \cdot \pi_{1}(A)=\pi(A) \cdot V \quad \text { for } \quad A \in \mathfrak{U}\left(V_{+}\right),
$$

and using the invariance of $\pi$ and $\pi_{1}$ under translations we get $\left\|\pi_{1}(A)\right\|=\|\pi(A)\|$ for each $A \in \mathfrak{A}$. Thus we may consider $\pi_{1}$ as a representation of $\pi(\mathfrak{H})$, and we can 
drop in the following the symbol $\pi$. Then we proceed in analogy to [2]: first we replace $\left(\pi_{1}, \mathscr{H}_{1}\right)$ by some equivalent representation $\gamma$ acting on $\mathscr{H}$,

$$
\gamma(A)=V \cdot \pi_{1}(A) \cdot V^{-1} \quad \text { for } \quad A \in \mathfrak{A} .
$$

From relation (5.1) we get

$$
\gamma(A)=A \quad \text { if } \quad A \in \mathfrak{A}\left(V_{+}\right),
$$

and the invariance of $\pi_{1}$ under translations implies that

$$
\gamma \alpha_{x}(A)=U_{\gamma}(x) \cdot \gamma(A) \cdot U_{\gamma}(x)^{-1}
$$

where $x \rightarrow U_{\gamma}(x)$ is some continuous unitary representation of the translations on $\mathscr{H}$. Then we define a transported representation ${ }^{x} \gamma$ of $\mathfrak{A}$ by

$$
x_{\gamma}(A)=\Gamma(x)^{-1} \cdot \gamma(A) \cdot \Gamma(x) \text { for } A \in \mathfrak{A},
$$

where $\Gamma(x)=U_{\gamma}(x) \cdot U(x)^{-1}$, and $U(x)$ denotes the translation implementing $\alpha_{x}$. It is obvious that the unitaries $\Gamma(x)$ satisfy the cocycle equation

$$
\Gamma(x+y)=\Gamma(x) \cdot \alpha_{x}(\Gamma(y))
$$

and that $x \rightarrow \Gamma(x)$ is strongly continuous. Moreover, it follows from relations (5.3) to (5.5) that ${ }^{x} \gamma(A)=A$ if $A \in \mathfrak{U}\left(V_{+}+x\right)$, and consequently

$$
\Gamma(x) \in \mathfrak{U}\left(V_{+}\right)^{\prime} \cap \mathfrak{U}\left(V_{+}+x\right)^{\prime} .
$$

So for each representation $\gamma$ with the properties (5.3) and (5.4) one can construct a cocycle $\Gamma$ satisfying (5.6) and (5.7). Conversely, given $\Gamma$ it follows from (5.5) that one can reconstruct $\gamma$ by

$$
\gamma(A)=n-\lim _{x} \Gamma(x) \cdot A \cdot \Gamma(x)^{-1}, A \in \mathfrak{A},
$$

where $x$ tends to the infinite past, i.e. $x_{0}+|\mathbf{x}| \rightarrow-\infty$.

Let us now assume that weak asymptotic completeness holds for the representation $\pi$. Then $\mathfrak{U}\left(V_{+}\right)^{\prime}=\mathfrak{F}^{\text {out }}\left(V_{-}\right)^{-}$, hence the cocycle $\Gamma$ has values in the algebra $\bigcup_{a} \mathfrak{F}^{\text {out }}\left(V_{-}+a\right)^{-}$generated by the outgoing free electromagnetic field. This means, in view of relation (5.8), that all states in the representation $\gamma$ can be obtained from the states in the representation $\pi$ by changing their outgoing radiation fields. We summarize these results in the following

Proposition 5.1. Let $(\pi, \mathscr{H})$ be an infrared minimal representation for which weak asymptotic completeness holds. Then each strongly continuous, unitary cocycle $\Gamma$ on $\mathscr{H}$ for which

i) $\Gamma(x+y)=\Gamma(x) \cdot \alpha_{x}(\Gamma(y))$

ii) $x \rightarrow U_{\gamma}(x) \equiv \Gamma(x) \cdot U(x)$ fulfills the spectrum condition,

iii) $\Gamma(x) \in \mathfrak{F}^{\text {out }}\left(V_{-}\right)^{-}$if $x \in V_{-}$

induces a representation $\gamma \in[\pi]$ given by

iv) $\gamma(A)=n-\lim _{x} \Gamma(x) \cdot A \cdot \Gamma(x)^{-1}, A \in \mathfrak{A}$

for $x$ tending to the infinite past. In fact, each representation $\pi_{1} \in[\pi]$ is equivalent to a representation $\gamma$ obtained this way. 
We remark that this result can be used to give a characterization of the superselection sectors belonging to $[\pi]$ in terms of the cohomology-classes of the cocycles $\Gamma^{8}$.

In conclusion we want to demonstrate that there exist uncountably many disjoint irreducible physical representations carrying a given electric charge. This substantiates the statements made in the Introduction. To simplify the discussion we will restrict our attention to representations of zero electric charge which are induced by the well-known asymptotic coherent photon states. (So these states can be defined on the algebra of the interacting electromagnetic field.) For the construction of these representations we will use the formalism developed above and exhibit suitable cocycles $\Gamma_{0}$ in the vacuum representation $\left(\pi_{0}, \mathscr{H}_{0}\right)$.

Let $F^{\text {out }}$ be any outgoing free electromagnetic field-operator which is affiliated to $\mathfrak{F}^{\text {out }}\left(V_{-}\right)^{-}$. Then for each $\varepsilon>0$

$$
W_{\varepsilon}=e^{i \int_{0}^{\infty} d t e^{-\varepsilon t \cdot \alpha-t}\left(F^{\text {out }}\right)} \in \mathfrak{F}^{\text {out }}\left(V_{-}\right)^{-}
$$

is a unitary operator, and one can define the cocycle

$$
\Gamma_{\varepsilon}(x)=W_{\varepsilon} \cdot \alpha_{x}\left(W_{\varepsilon}^{-1}\right) \in \mathfrak{F}^{\text {out }}\left(V_{-}\right)^{-} \cup \mathfrak{F}^{\text {out }}\left(V_{-}+x\right)^{-},
$$

which is clearly unitary and strongly continuous in $x$. It is easy to verify that the corresponding representation $\gamma_{\varepsilon}$ is given by

$$
\gamma_{\varepsilon}(A)=W_{\varepsilon} \cdot A \cdot W_{\varepsilon}^{-1} \text { for } A \in \mathfrak{U},
$$

hence it is unitarily equivalent to the vacuum representation. Yet if one proceeds to the limit $\varepsilon \rightarrow 0$ and chooses an operator $F^{\text {out }}$ such that $\left\|(H-i \varepsilon \cdot 1)^{-1} \cdot F^{\text {out }} \Omega\right\|$ tends to infinity (which implies that $\left(\Omega, W_{\varepsilon} \Omega\right)$ tends to zero), then the resulting representation $\gamma_{0}$ turns out to be disjoint from the vacuum representation.

For the proof establishing the existence of $\gamma_{0}$ the following remarks may suffice: since $F^{\text {out }}$ is a free field one can show by an explicit calculation that for each $x \in \mathbb{R}^{4}$ the limit $s$ - $\lim _{\varepsilon \rightarrow 0} \Gamma_{\varepsilon}(x) \Omega$ exists, the convergence being uniform on bounded regions of $\mathbb{R}^{4}$. Taking also into account that $\Gamma_{\varepsilon}(x) \cdot A \Omega=A \cdot \Gamma_{\varepsilon}(x) \Omega$ for $A \in \mathfrak{U}\left(V_{+}\right) \cap \mathfrak{U}\left(V_{+}+x\right)$ (because of Huygens' principle) as well as $\left\|\Gamma_{\varepsilon}(x)\right\|=1$ it then follows that

$$
s-\lim _{\varepsilon \rightarrow 0} \Gamma_{\varepsilon}(x)=\Gamma_{0}(x)
$$

exists. Hence $\Gamma_{0}$ is a continuous, unitary cocycle, and since $\Gamma_{0}(x) \in \mathfrak{F}^{\text {out }}\left(V_{-}\right)^{-}$if $x \in V_{\text {_ }}$ one can define

$$
\gamma_{0}(A)=n-\lim _{x} \Gamma_{0}(x) \cdot A \cdot \Gamma_{0}(x)^{-1}, A \in \mathfrak{U}
$$

for $x$ tending to the infinite past, which is the desired representation.

It follows from the cocycle equation for $\Gamma_{0}$ and the defining relation (5.13) that the translations in the representation $\gamma_{0}$ are given by $U_{0}(x)=\Gamma_{0}(x) \cdot U(x)$ where

$8 \quad \Gamma$ and $\Gamma^{\prime}$ belong to the same cohomology-class if $\Gamma^{\prime}(x)=V \cdot \Gamma(x) \cdot \alpha_{x}\left(V^{-1}\right)$ for some unitary $V \in \mathfrak{F}^{\text {out }}\left(V_{-}\right)^{-}$. The corresponding representations $\gamma$ and $\gamma^{\prime}$ are then equivalent 
$U(x)$ are the translations in the vacuum representation. Since $\Gamma_{0}$ is continuous it is also clear that $x \rightarrow U_{0}(x)$ is continuous, and using the fact that the spectrum condition holds for $U(x)$ and that

$$
U_{0}(x)=s-\lim _{\varepsilon \rightarrow 0} \Gamma_{\varepsilon}(x) \cdot U(x)=s-\lim _{\varepsilon \rightarrow 0} W_{\varepsilon} U(x) W_{\varepsilon}^{-1},
$$

one finds that $\operatorname{sp} \mathscr{U}_{0} \subset \bar{V}_{+}$.

In order to verify that $\gamma_{0}$ is irreducible one proceeds as follows: first one extends $\gamma_{0}$ by continuity to the algebra $\mathfrak{F}^{\text {out }}$ generated by the outgoing electromagnetic field. Using the $c$-number commutation relations for these fields it then follows that for each field $F^{\text {out }}$ which is affiliated to $\mathfrak{F}^{\text {out }}(\mathcal{O})$

$$
\gamma_{0}\left(e^{i F^{\prime \text { out }}}\right)=\zeta^{\prime} \cdot e^{i F^{\prime \text { out }}}
$$

where

$$
\zeta^{\prime}=\lim _{\varepsilon \rightarrow 0} e^{\left(\int_{0}^{\infty} d t e^{-\varepsilon t \cdot\left(\Omega,\left[F^{\prime \text { out }}, \alpha_{-t}\left(F^{\text {out }}\right)\right] \Omega\right)}\right)}
$$

is a phase-factor. Hence $\gamma_{0}$ acts like a coherent state representation on the outgoing electromagnetic fields, so in particular $\gamma_{0}\left(\mathfrak{F}^{\text {out }}(\mathcal{O})\right)=\mathfrak{F}^{\text {out }}(\mathcal{O})$ for each bounded region $\mathcal{O}$. From this one gets $\gamma_{0}(\mathfrak{U})^{-} \supset \mathfrak{F}^{\text {out }-}$, and since $\gamma_{0}\left(\mathfrak{U}\left(V_{+}\right)\right)=\mathfrak{U}\left(V_{+}\right)$ one arrives at

$$
\gamma_{0}(\mathfrak{A})^{\prime} \subset \mathfrak{U}\left(V_{+}\right)^{\prime} \cap \mathfrak{F}^{\text {out }}\left(V_{-}\right)^{\prime} .
$$

It then follows from the weak asymptotic completeness of the vacuum representation (Proposition 4.2) and the fact that $\mathfrak{U}\left(V_{+}\right)^{-}$is a factor that $\gamma_{0}$ is irreducible.

Now if $\gamma_{0}$ would be equivalent to the vacuum representation there would exist a unitary operator $W_{0} \in \mathfrak{F}^{\text {out }}\left(V_{-}\right)^{-}$such that $\gamma_{0}(A)=W_{0} A W_{0}^{-1}$ for $A \in \mathfrak{A}$. [This follows again from weak asymptotic completeness and the fact that $\gamma_{0}(A)=A$ if $\left.A \in \mathfrak{U}\left(V_{+}\right).\right]$As a consequence the vacuum state $\omega_{0}$ and the coherent state $\omega_{0} \circ \gamma_{0}$ would both induce the Fock-representation of the algebra $\mathfrak{F}^{\text {out }}$ [12]. But using relation (5.15) and (5.16) one can exclude this possibility if $\left\|(H-i \varepsilon \cdot 1)^{-1} \cdot F^{\text {out }} \Omega\right\|$ tends to infinity for $\varepsilon \rightarrow 0$ [23], hence with such a choice of $F^{\text {out }}$ the representation $\gamma_{0}$ is disjoint from the vacuum representation. By a similar argument one can also show that two representations $\gamma_{0}$ and $\gamma_{0}^{\prime}$, say, are disjoint if they are built from operators $F^{\text {out }}$ and $F^{\text {out }}$, respectively, for which $\left\|(H-i \varepsilon \cdot 1)^{-1} \cdot\left(F^{\text {out }}-F^{\prime \text { out }}\right) \Omega\right\|$ tends to infinity if $\varepsilon \rightarrow 0$. It is then clear that there exist uncountably many disjoint representations in the charge-class of the vacuum representation.

\section{Conclusions}

Using the concept of charge-classes we have characterized a set of states which seems to be appropriate for a general discussion of the structure of quantum electrodynamics. In contrast to the conventional field-theoretic settings, where one selects in some arbitrary way a separable space of states, we have taken into account an uncountable number of superselection sectors. As was demonstrated, 
this enlargement of the state-space has conceptual as well as technical advantages. In particular, one gains some flexibility in choosing the localization properties of charged states.

Amongst the various questions which have not been answered in this analysis let us mention the two perhaps most interesting ones : first, it would be desirable to understand better the structure of the asymptotic observables $\mathfrak{M}^{\text {in }}$ and $\mathfrak{M}^{\text {out }}$ which are associated with the massive particles. This would help to clarify the question as to which concepts are appropriate for the description of charged particles at asymptotic times [22]. As was indicated, the solution to this problem seems to depend only on Gauss' law and not on the detailed dynamical structure of quantum electrodynamics.

The second question which deserves further study is related to the concept of charge-classes. In order to see that the labels of these classes have, as expected, the meaning of a charge, one should show that there exists a composition law of charge-classes (charges can be added) and that to each charge-class there exists a conjugate class (to each charge there exists an opposite charge). A solution of this problem would generalize the fundamental results of Doplicher et al. [2] (see also [17]) on the superselection structure of theories with short-range forces to the case of quantum electrodynamics.

Acknowledgement. I am deeply indebted to Rudolf Haag for having turned my attention to the conceptual problems in quantum electrodynamics many years ago, and for his constructive criticism and constant support of my work. I have benefitted from discussions with Sergio Doplicher, Volker Enß, Klaus Fredenhagen, Jürg Fröhlich, and Eyvind Wichmann which have greatly influenced my present understanding of the subject. I would also like to thank Daniel Kastler for a critical reading of the manuscript. Finally, I am grateful for the hospitality extended to me at various stages of this work by the Department of Physics of the University of California, Berkeley, the Istituto Matematico dell' Università di Roma and the Centre Universitaire de Marseille-Luminy, as well as for financial support from the Max-Kade-Foundation, the CNR of Italy and the CNRS of France.

\section{References}

1. Jauch, J.M., Rohrlich, F.: The theory of photons and electrons. Berlin, Heidelberg, New York: Springer 1976

2. Doplicher, S., Haag, R., Roberts, J.E. : Local observables and particle statistics. I. Commun. Math. Phys. 23, 199 (1971)

3. Strocchi, F., Wightman, A.S.: Proof of the charge superselection rule in local relativistic quantum field theory. J. Math. Phys. 15, 2198 (1974)

4. Fröhlich, J., Morchio, G., Strocchi, F.: Infrared problem and spontaneous breaking of the Lorentz group in QED. Phys. Lett. 89B, 61 (1979)

5. Symanzik, K.: Lectures on Lagrangian quantum field theory. DESYT-71

6. Haag, R., Kastler, D.: An algebraic approach to field theory. J. Math. Phys. 5, 848 (1964)

7. Borchers, H.J., Buchholz, D.: To be published. For a sketch of the proof see [17]

8. Bisognano, J.J., Wichmann, E. : On the duality condition for a hermitean scalar field. J. Math. Phys. 16, 985 (1975)

9. Haag, R.: Lille Conference 1957 «Les problèmes mathématiques de la théorie quantique des champs». Paris: Èditions du CNRS 1959

10. Borchers, H.J.: Energy and momentum as observables in quantum field theory. Commun. Math. Phys. 2, 49 (1966)

11. Sakai, S.: $C^{*}$-algebras and $W^{*}$-algebras. Berlin, Heidelberg, New York: Springer 1971

12. Buchholz, D.: Collision theory for massless Bosons. Commun. Math. Phys. 52, 147 (1977) 
13. Borchers, H.J.: A remark on a theorem of B. Misra. Commun. Math. Phys. 4, 315 (1967)

14. Borchers, H.J.: On the converse of the Reeh-Schlieder theorem. Commun. Math. Phys. 10, 269 (1968)

15. Borchers, H.J.: On groups of automorphisms with semi-bounded spectrum. Paris: Èditions du CNRS No. 181,1970

16. Sadowski, P., Woronowicz, S.L.: Total sets in quantum field theory. Rep. Math. Phys. 2, 113 (1971)

17. Buchholz, D., Fredenhagen, K. : Locality and the structure of particle states. Commun. Math. Phys. 84, 1-54 (1982)

18. Kraus, K., Polley, L., Reents, G.: Models for infrared dynamics. I. Classical currents. Ann. Inst. H.Poincaré 26, 109 (1977)

19. Schroer, B.: Infrateilchen in der Quantenfeldtheorie. Fortschr. Physik 11, 1 (1963)

20. Araki, H., Haag, R.: Collision cross sections in terms of local observables. Commun. Math. Phys. 4, 77 (1967)

21. Buchholz, D.: In: "Proceedings of the international conference on operator algebras, ideals and their applications in theoretical physics, Leipzig 1977". Leipzig: Teubner 1978

22. Fröhlich, J.: On the infrared problem in a model of scalar electrons and massless scalar Bosons. Ann. Inst. H.Poincaré 19, 1 (1973)

Fröhlich, J., Morchio, G., Strocchi, F.: Charged sectors and scattering states in quantum electrodynamics. Ann. Phys. 119, 241 (1979)

23. Roepstorff, G.: Coherent photon states and spectral condition. Commun. Math. Phys. 19, 301 (1970)

24. Gervais, J.L., Zwanziger, D. : Derivation from first principles of the infrared structure of quantum electrodynamics. Phys. Lett. 94B, 389 (1980)

Communicated by R. Jost

Received January 15, 1982 
